\title{
Erratum: Quench-Induced Breathing Mode of One-Dimensional Bose Gases [Phys. Rev. Lett. 113, 035301 (2014)]
}

Bess Fang, Giuseppe Carleo, Aisling Johnson, and Isabelle Bouchoule

(Received 25 March 2016; published 21 April 2016)

DOI: 10.1103/PhysRevLett.116.169901

There is a typographical error in an inline equation on p. 2, right-hand column, in the ninth line following Eq. (1). The equation for the momentum distribution should read $n(p, \tau) \propto \rho_{0}(p /(\dot{b} m))$. This does not impact the results of the Letter, as the simulation depicted in Figs. 1(e) $-1(\mathrm{~g})$ has been performed with the correct expression. 